QVIPURAMAYOC Revista de la Facultad de Ciencias Contables

Vol. 17 N. ${ }^{\circ} 33$ pp. 97-106 (2010) UNMSM, Lima, Perú

ISSN: 1560-9103 (versión impresa) / ISSN: 1609-8196 (versión electrónica)

\title{
COMPETITIVIDAD Y PLAN INDUSTRIAL NACIONAL
}

COMPETITIVENESS AND INDUSTRIAL NATIONAL PLAN

\section{Adrián Alejandro Flores Konja *}

Docente Principal de la Facultad de Ciencias Contables, UNMSM

\section{Manuel Alberto Hidalgo Tupia ${ }^{* *}$}

Docente de la Facultad de Ciencias Contables, UNMSM

[Recepción: Enero de 2010 / Conformidad: Marzo de 2010]

\section{RESUMEN}

El presente artículo, que reseña una investigación exploratoria, analiza los conceptos del Plan Nacional de Competitividad, propuesto por el Consejo Nacional de Competitividad, determinando que no existe en él un Plan Industrial explícito, que defina el lugar del Perú en la división internacional del trabajo, distinto del "Plan" de facto, que somete al Perú a la producción de minerales y productos agrícolas, o servicios turísticos, sin incursionar en la industria. Analiza la especialización del Perú en minerales sin procesar y otros productos extractivos primarios, y sus consecuencias. El artículo analiza también cómo se han formulado los Proyectos Nacionales en la historia republicana, en relación al Plan Industrial, y da una valoración de dichos esfuerzos fallidos. Señala también los requisitos en infraestructura, educación y ciencia y financiamiento que se requieren para llevar a la práctica un verdadero Plan Industrial.

Se propone, por último, algunos de los elementos que pueden incluirse en el Plan Industrial, de acuerdo a la vocación física del país y las demandas del desarrollo científico y tecnológico de la humanidad.

Palabras clave: Competitividad, Plan Industrial, Industrialización, MYPE, Estado. Infraestructura, Educación, Financiamiento, Exportación, Consejo Nacional de Competitividad, Plan Nacional de Competitividad.

\section{ABSTRACT}

This article, which discusses an exploratory investigation analyses the concepts of the National Competitiveness Plan, proposed by the National Competitiveness Council, determining that there is in it an explicit Industrial Plan, which defines the location of Peru in the international division of work different than the "Plan" de facto subjecting Peru to the production of minerals and agricultural products, or tourism, without getting into the industry. Analyse Peru's specialization in raw minerals and other primary extractive products, and their consequences. The article analizes how was formulated the National Projects in the republican history too in relation to the industrial plan and gives an assessment of why theses effort failed. It also notices the requirements in infrastructure, education and science funding needed to implement a real industrial plan.

Finally. it proposes some elements that can be included in the Industrial Plan, according to the country's natural vocation and the demands of scientific and technological development of mankind.

Keywords: Competitiveness, Industrial Plan, Industrialization, MSEs, State, Infrastructure, Education, Funding, Export, CNC, PNC.

\footnotetext{
* Doctor en Ciencias Contables y Empresariales, UNMSM. Contador Público Colegiado Certificado, actual Decano de la Facultad de Ciencias Contables, UNMSM. E-mail: afloreskonja@yahoo.es

** Economista, UNMSM. Docente de la Facultad de Ciencias Contables, UNMSM. E-mail: mhidalgot@hotmail.com
} 


\section{INTRODUCCIÓN}

El presente artículo es el resultado de una investigación exploratoria bibliográfica sobre la competitividad, y las políticas publicas al respecto que le van a dar a las MYPEs y a las demás empresas la sinergia para lograr una competitividad individual en el mercado local e internacional. La inquietud surgió durante la investigación sobre los Mecanismos de Promoción de las Exportaciones para las MYPE, que nos permitió ver que sin un compromiso del Estado en mejorar la Competitividad global de la economía, las políticas de promoción de exportaciones caían en un saco roto.

Las políticas de competitividad expuestas por el Consejo Nacional de Competitividad, CNC, por ejemplo, en el Plan Nacional de Competitividad (PNC), a nuestro parecer, adolecen de un formalismo fuera de contexto, propio de un libro de texto. Además de su imprecisión, carece de lo fundamental: una visión prospectivista, "voluntarista", que le dé un papel a la voluntad nacional de desarrollarse; más allá de la creencia ingenua en la "mano invisible" del "libre mercado". Es decir, el concepto que sólo la intervención voluntaria del poder nacional puede generar un proceso de desarrollo industrial en el cual la libre iniciativa de los emprendedores sea el motor principal, pero con la "chispa de encendido" y la "generación del clima" desarrollista que sólo puede aportar la sociedad organizada (Estado).

La carencia mayor del CNC y el PNC es el de un Plan Industrial, explícito, que es el direccionamiento a los productos que el país elegirá para su especialización, y los pasos que va a dar para lograr así la competitividad en los sectores o nichos elegidos.

En realidad, en el Perú existe un "Plan Industrial”, pero que es retrógrado y sumamen- te inconveniente para el país, razón por la cual solo se hace explícito tímida y parcialmente, pero se aplica en la práctica. El lugar del Perú en la división internacional del trabajo es: producción de minerales e hidrocarburos y productos agrícolas, sin dejar de mencionar las exportaciones ilegales de drogas, que han dado al Perú la imagen de un país problema en la escena regional y mundial, como Colombia y, actualmente, México. El "Plan Industrial” implícito vigente excluye sistemáticamente la producción manufacturera, que representa una mayor complejización organizacional, técnica y científica, más acorde con la evolución de la especie humana. Estas omisiones hacen ver una "voluntad" aplicada por algunos sectores locales y globales contra el Estado peruano para que no se aplique un verdadero Plan Industrial, cuyos costos y resultados pueden alterar un estado de cosas muy conveniente para dichos sectores.

El presente artículo concluye esbozando algunos elementos vitales para un Plan Industrial, y algunos requisitos y condiciones para el mismo, en relación a la infraestructura, educación y ciencia, y financiamiento.

\section{DESARROLLO, INDUSTRIALIZACIÓNY COMPETITIVIDAD}

El concepto de desarrollo, unido al de industrialización, era el concepto dominante en los siglos XIX y XX y lo es en la actualidad para los países del Asía Pacífico, y tiene como paradigma la Revolución Industrial en Inglaterra y los procesos posteriores de industrialización, el primero de ellos, de los Estados Unidos de América, Francia y Alemania.

El desarrollo, mas allá que el mero crecimiento del producto nacional - PBI, implica 
un proceso de complejización productiva de la economía nacional. Esta complejización se asoció siempre con la industrialización, cuyos elementos eran la producción fabril y en masa (con el aprovechamiento de las economías de escala), el maquinismo y la introducción acelerada de la tecnología y la innovación a través de las máquinas herramientas $\mathrm{y}$ los propios procesos industriales.

La industrialización se asociaba a la creación de un mercado nacional extenso, (arrinconando y hasta suprimiendo la economía natural -de subsistencia- y artesanal) y con un proceso de acumulación de capital por una clase capitalista en formación y consolidación. Además, el requisito era la creación de infraestructura, para posibilitar un mercado accesible, el abasto oportuno y económico de materias primas y demás recursos, energía, conducción de aguas, irrigaciones, etc. Además, se requería una educación reformada acorde a las nuevas necesidades. Los paradigmas de desarrollo industrial fueron los ejemplos de Gran Bretaña, EE.UU., Francia, Alemania, Japón, y mas contemporáneamente, después de la Segunda Guerra, Corea, Taiwán y las gigantes China e India. En nuestra región, tenemos los casos de desarrollo truncados como Brasil, Argentina y México. Este paradigma es originalmente de un "desarrollo hacia adentro" que pronto se revertía "hacia fuera", como en los casos mas recientes.

En America Latina, la CEPAL produjo una versión teórica llamada Industrialización por sustitución de Importaciones, ISI, que desde un punto de vista monetarista ("ahorro de divisas") se enfocó en el aspecto comercial y no en el desarrollo de las fuerzas productivas, propiamente. Esta versión se aplicó paralelamente a la creación de infraestructura; hasta inicios de los años
80 , en que se produce la crisis de la deuda de América Latina y la capacidad financiera de nuestros gobiernos se desploma. La ISI propició una industrialización "light" de artículos de consumo directo, creando industrias de ensamblaje de automotores electrodomésticos, etc. que en nuestro país desaparecieron ante los primeros embates de la apertura comercial de Ulloa, Boloña Behr y demás. Los desarrollos más serios corrieron la misma suerte.

La Competitividad es en realidad un concepto subordinado, en el contexto de la globalización y la apertura nos habla del desempeño de las economías en el mercado mundial, esto, tal como lo define el Foro Económico Mundial FEM). Este, en colaboración con el Instituto de Desarrollo Internacional de la Universidad de Harvard, elabora un Informe Global de la Competitividad, donde se define la Competitividad como "la capacidad que tiene un país para lograr altas tasas de crecimiento", lo que implica "desarrollar un "clima" económico, político y social que le permite incrementar la productividad de los factores de producción" [Plan Nacional de Competitividad] .

Nótese que el FEM usa la palabra crecimiento en vez de desarrollo, como si fueran sinónimos.

El PNC dice que: "El Perú se encuentra en un proceso de apertura comercial" porque considera que la liberalización comercial permite a los diversos sectores de la economía alcanzar un mayor desarrollo como resultado del acceso a mercados ampliados y un crecimiento de los niveles de inversión productiva.

El PNC presenta 7 objetivos estratégicos que son indispensables, pero pierden como sistema al no definir la estructura industrial deseable para el país, y el financiamiento del desarrollo. 


\section{Los objetivos estratégicos son:}

1. Fortalecimiento institucional

2. Política económica, mercados financieros y de capitales

3. Infraestructura

4. Articulación empresarial (cadenas productivas)

5. Innovación tecnológica e innovación empresarial

6. Educación

7. Medio Ambiente

Las políticas de promover la Competitividad, como se aplican en las naciones industrializadas, inciden en la investigación e innovación científicas, en una articulación de 3 sectores: las empresas, el gobierno y las universidades e institutos de investigación, como se aprecia para el caso de Finlandia en el Cuadro N.o 1.

\section{Perfil industrial y del comercio exterior peruano}

Si se analiza los principales productos de exportación del Perú; descubrimos que son mayormente minerales; petróleo; productos extractivos pesqueros, productos agrícolas; y solo en un lugar relegado, productos manufacturados como confecciones y textiles. Es decir, productivamente somos un país campo-mina. La mínima complejización industrial lograda con la ISI (Producción de línea blanca y electrodomésticos, automóviles, etc.) se destruyó con la apertura de los años ochenta y noventa.

En productos manufacturados, el Perú logró una cierta especialización a partir de su riqueza extractiva metal mecánica; productos eléctricos (conductores, etc.) construcción naval, carrocerías de automóviles, etc.

Además, el Perú, al formar el mercado ampliado andino, logró ser favorecido por algunas transnacionales para su producción regional de productos de limpieza y aseo personal: perfumería, alimentos procesados, etc; pero en un nivel menor a Colombia y Chile, por ejemplo.

En los demás bienes de consumo; los productos agropecuarios, alimentos procesados, productos de limpieza aseo personal, cemento, fierro y demás productos para la construcción, son los más destacados.

Gráfico N.o 1. La triple hélice.

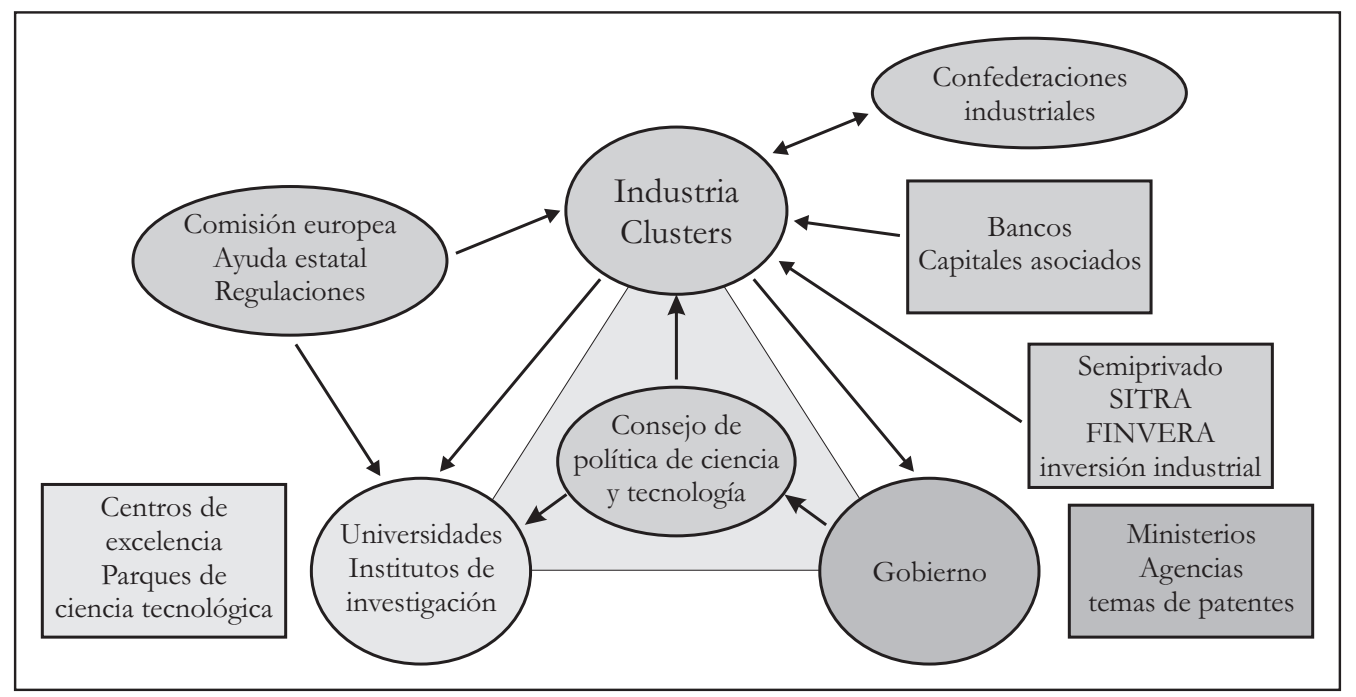

Fuente: Dr. Heikki Kotilainen, I Foro Nacional de Ciencia, Tecnología, Innovación y Competitivad. Lima, abril 9, 2008. 
Las exportaciones tuvieron un crecimiento del 43\% entre el 2004 y 2008; que ha dependido básicamente del precio de los minerales, un resultado buscado por los diseñadores de la política económica, y han justificado un concepto de "crecimiento para afuera”, en la que las exportaciones son la locomotora del crecimiento del PBI. Pero este "Crecimiento hacia afuera" es un mal sustituto del desarrollo industrial, aunque en ocasiones parezca bueno, pues ha creado un ciclo económico de expansión ficticia y grandes recesiones, al compás de la volatilidad de los mercados mundiales. Por ejemplo, una coyuntura de bajos precios de los minerales crea, como en 1998, una recesión económica acentuada.

El "Crecimiento hacia fuera" se sostiene en dos fuentes "extraordinarias": las remesas de efectivo de los cientos de peruanos en el exterior; y las "exportaciones" ilegales de estupefacientes, que crean un "colchón" de divisas que evita una crisis de abastecimiento y una mayor crisis social, pero a costa de socavar la seguridad interna nacional.

Michael Porter, "gurú" de la Competitividad, en su reciente visita al Perú, para exponer el 30 de noviembre de 2009 en el Seminario "Claves para una estrategia competitiva”, organizado por la Universidad del Pacífico, se refirió al perfil industrial y del comercio exterior peruano en forma muy clara, y revolvió el ambiente con sus declaraciones. La primera es que el Perú carece de una política de largo plazo en materia de competitividad, y que la economía peruana no tiene un rumbo definido.

Además, dijo que "los inversionistas extranjeros que quieren construir fábricas no están pensando en el Perú". Afirmó que "Perú ha crecido mucho en los últimos años, pero ese crecimiento no se ha reflejado en beneficio de la mayoría de la población”, añadiendo que hemos "vivido una ilusión exportadora en los últimos años"; lo que se debería a que "el Perú no dispone de una política ni de una estrategia de largo plazo en temas de competitividad y, por tanto, es una economía que no tiene un rumbo definido".

Es más, continúa el profesor de Harvard: "Perú tendrá que hacer más que solamente exportar materias primas, porque de lo contrario, hay riesgo de que China congele a la economía peruana en su forma actual"; es decir, como simple depósito de materias primas, cuando deberíamos centrarnos en "las exportaciones de productos con valor agregado y de servicios, que casi se han estancado"

En realidad, la evidencia empírica es que hemos pasado por sucesivas "eras" o "booms" de exportación, con la sucesión del oro, guano, salitre, petróleo, caucho, azúcar, algodón, cobre, nuevamente oro, gas, eras

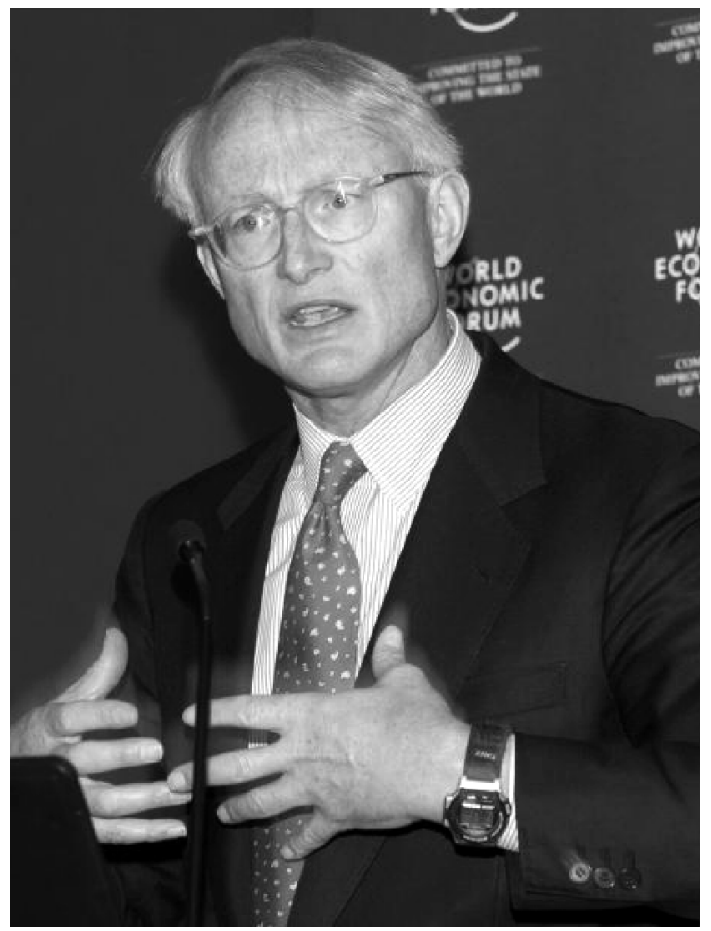

Michael Porter. "Gurú” de la Competitividad. En su reciente visita a Lima, para exponer el 30 de noviembre de 2009 en un Foro de la Universidad del Pacifico, revolvió el ambiente con sus declaraciones. 
que terminan sin dejar nada duradero en la economía, es decir, sin capitalizar al país. Como se puede apreciar, las exportaciones no tradicionales, que empezaron a tomar importancia con la era del desarrollismo, en la actualidad empiezan a perder su importancia relativa, avasallada por los minerales y sus altos precios de coyuntura.

Por ejemplo, en el cuadro N. ${ }^{\circ} 1$ se muestra los principales productos de exportación desde 1900, con sus periodos de vigencia.

Nótese que productos como el algodón, azúcar, caucho, lana, han tenido una vida efímera, siendo el producto más permanente el conjunto de los minerales. Los productos no tradicionales tuvieron su máximo a mediados de la década de 1990, pero están disminuyendo su importancia relativa.

\section{Los proyectos de desarrollo en el Perú}

Solo a partir de la década de 1840, el Perú pudo con Ramón Castilla, ordenar al Estado y obtener los medios para proponer un plan de desarrollo industrial "a la norteamericana", usando el guano y fallidamente el salitre como fuente financiera, y no como vocación exportadora permanente.
Fue la facción llamada Republicana "liberal" (como opuesta al monarquismo o colonialismo, diferente a su acepción en la actualidad) en especial, después de la Revolución Liberal de 1864, la que sentó las bases para un desarrollo basado en la expansión ferroviaria, producción manufacturera, especialmente textil, y en la agricultura intensiva de algodón y caña de azúcar, con proyecciones a otras áreas. La sucesión de gobiernos de Prado, Balta y Pardo, con el programa ferroviario, la organización de la educación básica pública, la creación de la escuela de Ingenieros y la Facultad de Ciencias y de Ciencias Económicas y comerciales de San Marcos, la creación del Cuerpo de Ingenieros; tenían la intención del desarrollo, en su parte inicial. Pardo, el más esclarecido exponente de esta facción, esboza en sus escritos elementos de un Plan Industrial, por ejemplo, proponiendo la instauración de la industria textil que transforme la lana, que se exportaba entonces en crudo, usando como ejemplo la ciudad industrial de Cincinatti, en Estados Unidos. (Mc Evoy, Op. Cit., p. 25-26). Pardo también aboga expresamente por transformar los ingresos por las exportaciones de productos extractivos, como el guano y el salitre, en infraestructura básica, como pre requisitos para la industrialización.

\section{Cuadro N.o 1}

\begin{tabular}{|l|l|l|l|l|l|l|l|l|l|l|}
\hline \multicolumn{1}{|c|}{ Producto } & 1910 & 1920 & 1930 & 1940 & 1950 & 1960 & 1970 & 1980 & 1990 & 2000 \\
\hline Minerales & & & & & & & & & & \\
\hline Algodón, azúcar, café & & & & & & & & & & \\
\hline Petróleo y derivados & & & & & & & & & & \\
\hline Harina de pescado & & & & & & & & & & \\
\hline Caucho, cuero y lana & & & & & & & & & & \\
\hline No tradicionales & & & & & & & & & & \\
\hline
\end{tabular}

Fuente: Acuña, Alexy y Zulueta, José. Crecimiento y ciclos económicos en el Perú, 1896 - 2008”. Lima. Universidad del Pacífico, noviembre 2009; p. 14 (inédito). Citado por Schultz, Jurgen, en "La Republica”. 


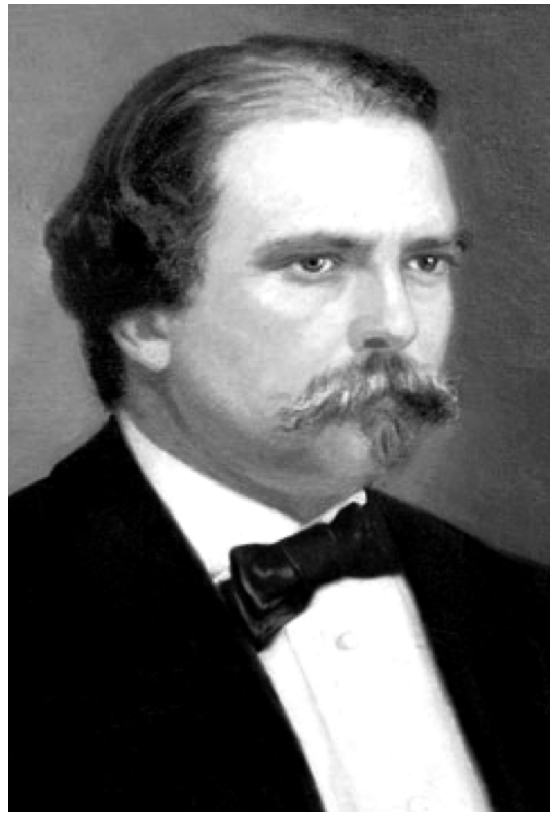

Manuel Pardo: Primer presidente civil del Perú, promotor de la Facultad de Ciencias de San Marcos y organizador de la educación básica pública. Promotor del proyecto ferroviario y desarrollista.

Este proceso fue desmantelado por la Guerra del Pacífico, originada cuando el Perú intentó tomar el salitre para financiar el proceso de construcción de infraestructura, luego que la Casa Dreyffus acabase con la fuente que provenía del guano.

El contrato Grace, con la apropiación de los acreedores de los principales activos del país; y la anulación de la capacidad financiera del Estado; postergó por décadas la reanudación del proceso de desarrollo. Fue el ilustre sanmarquino Santiago Antúnez de Mayolo el ideador de la nueva etapa desarrollista, enfocada en el desarrollo hidroeléctrico, de irrigaciones, industria siderúrgica y química, como ejemplifica la Corporación Peruana del Santa, en las décadas de 1940 al 1960, encargada de la primera hidroeléctrica moderna y el desarrollo siderúrgico en Chimbote, donde se planteaba también una industria electroquímica. Antúnez defendía la labor de la Corporación de sus detractores con sólidos

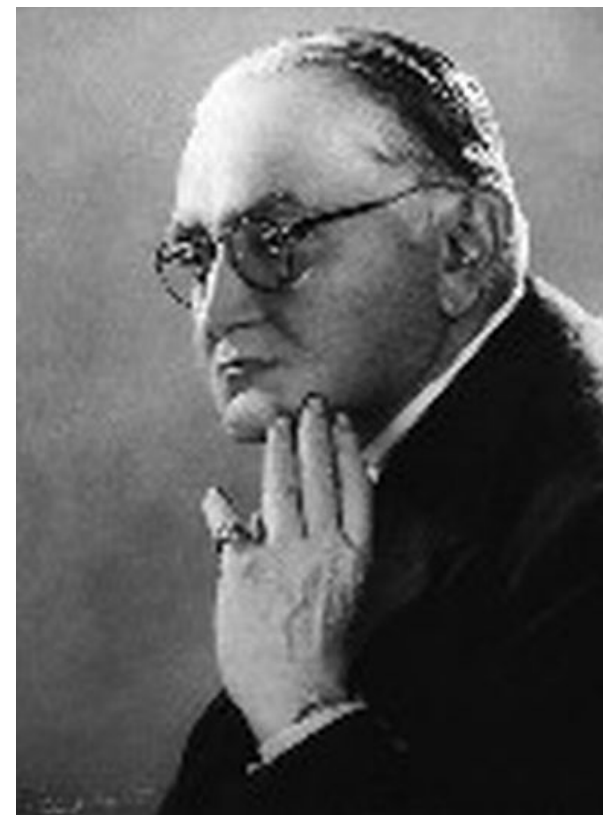

Santiago Antúnez de Mayolo, de la Facultad de Ciencias de San Marcos, promotor del Plan Eléctrico Nacional, que ideó la cascada de hidroeléctricas, junto con la siderurgia y la industria química.

argumentos: "Muchos piensan que el Perú debe seguir siendo un país de estructura económica colonial: agrícola y minera a base del 'Cholo barato'. Son estos los que propalan los malévolos comentarios sobre la inutilidad de las obras que realiza la Corporación”. Así, en el contexto de la post Guerra y el New Deal de Roosevelt, el país pudo replantear su desarrollo, en la época del "desarrollismo" latinoamericano, en los años 40 hasta la primera mitad de los 70, cuando se produce la primera crisis de la deuda, y los planes de infraestructura son cancelados.

Otro hito importante fue la integración económica a través del Pacto Andino de 1970 que planteó un Plan Industrial Andino, en el cual al Perú le tocó el desarrollo de motores carrocerías, material eléctrico, producción química básica, entre otros. Estos planes también tuvieron vida efímera, cuando la capacidad financiera de los Estados-miembros se desplomó con la crisis de la deuda. 


\section{Tendencias nacionales e internacionales}

Globalmente, las tendencias fundamentales que no dependen solo de las decisiones y políticas implantadas desde los cuarteles de la Organización para la Cooperación Económica y Desarrollo, del Fondo Monetario Internacional, o del Foro de Davos, son:

- Presencia de una moneda de cambio mundial, unida a la facilidad de transacciones financieras internacionales y de reducción del control del movimiento de capitales.

- Crecimiento de la Inversión Extranjera Directa.

- Expansión de las transacciones financieras-especulativas-como relación al comercio real.

- Expansión del Comercio Internacional.

- Incorporación acelerada de la ciencia y Tecnología en la construcción de máquinas-herramienta.

- Desarrollo de los países Asiáticos, en especial China, pero también Japón, Corea, Taiwán, etc., India y Rusia de la energía nuclear como fuente para el futuro.

- Desarrollos en los países asiáticos, de proyectos de alta tecnología en las áreas siguientes: Exploración espacial; telecomunicaciones; superconductores; transporte por levitación magnética; física de plasmas; biotecnología, aleaciones especiales, tecnología de láseres, óptica biofísica, etc.

En nuestro país, las tendencias son contradictorias con estas tendencias globales, siendo las principales:

- Mantención de la estructura productiva primaria - terciaria.

- Déficit creciente de infraestructura.

- Crecimiento demográfico moderado por la migración externa.
- Crecimiento del narcotráfico y su entroncamiento a la economía y vida social.

- Mantención de los niveles de pobreza y desigualdad

Es decir, nuestra "Política Industrial" informal alienta las tendencias entrópicas, negativas y al contrario de las tendencias desarrollistas modernas de los pases del Pacífico Asiático.

\section{Elementos a considerar en un plan indus- trial para el Perú}

Como muchos otros antes lo han señalado; la vocación productiva del Perú debe partir de su acervo natural, pero ir mucho más allá de él, en un propósito "voluntarista" o "prospectivo". Perú es un país cuya geología y topografía, definida por los portentosos Andes, y un clima signado por la Corriente de Humboldt, nos muestra una topografía accidentada, un gran acervo minero, del tamaño de un continente, $y$ una gran riqueza hidrobiológica. Debido a los Andes, el transporte se hace muy difícil, debiéndose crear un sistema complejo, como lo entendió Manuel Pardo ya en el siglo XIX. Los Andes brindan fuentes energéticas eficientes, como las caídas de agua para la formación de energía hidroeléctrica, y uranio, para producir energía nuclear.

¿Qué puede producir el Perú aparte de lo que ya produce?

Esta lista preliminar, necesariamente incompleta, deberá ser completada con el aporte de la comunidad científica:

* Aleaciones especiales y estratégicas. Lo que incluye la creación de una nueva refinación de minerales moderna

* Productos de metal mecánica y de conducción eléctrica, mejorando lo ya exis- 
tente con incorporación tecnológica masiva.

* Construcciones navales; realzando las capacidades existentes en el pasado en SIMA.

* Construcción y reparación de material rodante ferroviario, de preferencia, eléctrico.

* Dominio del proceso del uranio para generación eléctrica y otras aplicaciones de procesamiento de alimentos, de salud e industriales.

* Industrias petroquímicas, en base al gas de Camisea (plásticos, resinas, fibras sintéticas y fertilizantes).

* Electrónica básica, motores, refrigeración.

Como criterio general, el Perú no necesita incursionar en todos los sectores productivos, ni siquiera en todos los sectores modernos, debe concentrarse solo en aquellos en los que puede formar una ventaja competitiva que este bajo nuestro control. Hay áreas en que ningún país puede dejar de estar (electrónica; motores para transporte, etc.), y en otras el Perú aprovecha sus condiciones excepcionales.

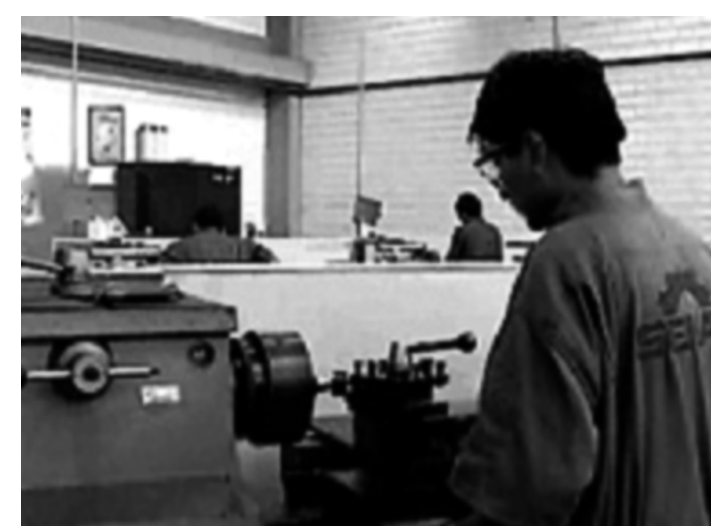

Enseñanza de operación de máquinas herramienta en una institución educativa nacional.
Este proceso debe comenzar con la creación de las Competencias científicas y tecnológicas necesarias, con la creación de institutos de investigación y enseñanza en metalurgia avanzada, energía nuclear, petroquímica, electrónica, construcción naval, diseños de máquinas eléctricas, entre otras.

El paso siguiente es brindar el apoyo estatal a las inversiones privadas que son de necesidad pública en esas áreas, garantizándoseles estabilidad, beneficios tributarios, apoyo tecnológico, créditos blandos y a bajas tasas con garantía pública, y un mercado interno protegido.

No es desapercibo para los autores que esto implica cambios de las políticas estatales y aún constitucionales, y un cambio total del "clima" generado por la globalización; pero, ¿Es realista considerar a la globalización como el imperio que durará mil años?

\section{CONCLUSIONES}

Sin un proceso de discusión nacional para la definición de un plan nacional industrial, con el compromiso respectivo y el consenso necesario, el país no podrá tener un desarrollo económico adecuado, acorde con las tendencias mundiales.

Sin este Plan nacional industrial, tampoco se podrá plantear una verdadera política de competitividad.

El Plan Nacional industrial debe relacionarse a la vocación física del país, pero no limitándose a ella, guiarse por el imperativo de ubicar al Perú en el comercio internacional y desarrollo científico de la humanidad.

El Plan Nacional industrial tiene requisitos y condiciones sin los cuales sería imposible de implementarse. 


\section{RECOMENDACIONES}

Debe abrirse la discusión sobre el Plan industrial Nacional, sus condiciones y requisitos, como un verdadero medio de consenso nacional en el contexto actual.

La comunidad universitaria debe comprometerse a iniciar el intercambio de ideas, por su papel central en el proceso del Plan Industrial y competitividad nacional.

El Instituto de Investigación de la Facultad de Ciencias Contables y Financieras debe asumir el liderazgo en la generación de este intercambio.

\section{REFERENCIAS BIBLIOGRÁFÍCAS}

1. Consejo Nacional de Competitividad. Plan Nacional de Competitividad: Buscando la inserción exitosa del Perú en el mercado global. (Síntesis). En CNC: http://www. perucompite.gob.pe (15/03/2010).
2. Diario Gestión (01 / 12 / 2009).

3. Hidalgo, Manuel. Santiago Antúnez de Mayolo: Visión de Estadista. En: Revista Red Eléctrica. Oct.-Nov., 2004, Lima.

4. Instituto Schiller: La Integración Iberoamericana. The New Benjamin Franklin House Publishing. Caracas, 1987.

5. Kotilainen, Heikki. Exposición en el I Foro NacionaldeCiencia,Tecnología,Innovación y Competitividad, Lima (09/04/2008).

6. Mc Evoy, Carmen. Pardo: Escritos Fundamentales. La huella Republicana Liberal en el Perú. Lima, 2004. Fondo Editorial del Congreso del Perú.

7. Porter, Michael. Competitividad una nueva estrategia económica para el Perú; presentación del 30/11/2009. En: http://www.isc.hbs.edu/ (consultado el 15/03/2010).

8. Porter, Michael. Competitive strategy (The free press, 1980).

9. Porter, Michael. Competitive advantage (The free press, 1985). 